\title{
The safety and efficacy of weekly paclitaxel administration for anaplastic thyroid cancer patients: A nationwide prospective study
}

\author{
Naoyoshi Onoda, ${ }^{1,2}$ Kiminori Sugino,,3 Takuya Higashiyama,,4 Makoto Kammori,,5 Kazuhisa Toda, \\ Ken-ichi Ito,,7 Akira Yoshida, ${ }^{1,8}$ Nobuyasu Suganuma, Noriaki Nakashima, ${ }^{10}$ Shinichi Suzuki,,11 \\ Kiyoaki Tsukahara, ${ }^{12}$ Hitoshi Noguchi, ${ }^{13}$ Masanori Koizumi, ${ }^{14}$ Toshimitsu Nemoto, ${ }^{15}$ \\ Hisato Hara, ${ }^{1,16}$ Akira Miyauchi, ${ }^{1,4}$ and Iwao Sugitani, ${ }^{1,6,17}$
}

Background: Anaplastic thyroid cancer (ATC) is a rare and extremely aggressive malignancy, with a median survival of less than 6 months due to rapid progression and resistance to multimodal therapies. Effective treatment strategies have not been identified. A prospective clinical study was performed to objectively evaluate outcomes of treatment with paclitaxel.

Methods: An investigator-initiated, multicenter, nonrandomized, open-label, single-arm study to evaluate the feasibility and efficacy of weekly paclitaxel $\left(80 \mathrm{mg} / \mathrm{m}^{2}\right)$ administration for patients with pathologically confirmed ATC was conducted in a nationwide organization.

Results: Feasibility was analyzed in 56 patients. More than one course of treatment was performed in 52 (93\%) patients retaining sufficient dose intensity $(>84 \%)$. No patient had to terminate the treatment because of an adverse event. The median overall survival was 6.7 months [confidence interval 4.4-9.0]. The 6-month survival was 54\%. Among the 42 patients with an evaluable lesion, none demonstrated complete remission, 9 (21\%) showed partial remission, $22(52 \%)$ achieved stable disease, and $8(19 \%)$ exhibited progressive disease; 3 did not complete the initial treatment course. The objective response rate was $21 \%$, and the clinical benefit rate was $73 \%$. The median time to progression was 1.6 months. Statistically, no additional effect of concomitant radiation was demonstrated in 6 patients receiving combined therapy. Eight patients, in whom a complete posttreatment surgical removal of the tumor was feasible, survived significantly longer (median 7.6 months [CI 8.123.0]) than the other 34 patients in whom the tumor could not be completely removed after chemotherapy (5.4 months [CI 3.0-7.8], $p=0.018$ ).

Summary: The study demonstrates objective and accurate information concerning the feasibility and efficacy of a standardized treatment with weekly paclitaxel administration for ATC patients.

Conclusions: Weekly paclitaxel administration for ATC patients can be of clinical benefit in a neo-adjuvant setting.

${ }^{1}$ Prospective Clinical Study Committee of Anaplastic Thyroid Carcinoma Research Consortium of Japan, Tokyo, Japan.

${ }^{2}$ Department of Surgical Oncology, Osaka City University Graduate School of Medicine, Osaka, Japan.

${ }^{3}$ Department of Surgery, Ito Hospital, Tokyo, Japan.

${ }^{4}$ Department of Surgery, Kuma Hospital, Kobe, Japan.

${ }^{5}$ Department of Surgery, Kanaji Thyroid Hospital, Tokyo, Japan

${ }^{6}$ Division of Head and Neck, Cancer Institute Hospital, Tokyo, Japan.

${ }^{7}$ Department of Surgery II, Shinshu University, Matsumoto, Japan.

${ }^{8}$ Department of Breast and Endocrine Surgery, Kanagawa Cancer Center, Yokohama, Japan.

${ }^{9}$ Department of Surgery, Yokohama City University, Kanagawa, Japan.

${ }^{10}$ Department of Breast and Endocrine Surgery, Tohoku University, Sendai, Japan.

${ }^{11}$ Department of Thyroid and Endocrinology, Fukushima Medical University School of Medicine, Fukushima, Japan.

${ }_{12}^{12}$ Department of Otolaryngology, Tokyo Medical University, Tokyo, Japan.

${ }^{13}$ Noguchi Thyroid Clinic and Hospital Foundation, Oita, Japan.

${ }^{14}$ Department of Surgery, Mito Medical Center, Mito, Japan.

${ }^{15}$ Department of Otorhinolaryngology, Japanese Red Cross Narita Hospital, Chiba, Japan.

${ }^{16}$ Department of Breast and Endocrine Surgery, Tsukuba University, Tsukuba, Japan.

${ }^{17}$ Department of Endocrine Surgery, Nippon Medical School, Tokyo, Japan. 


\section{Introduction}

A NAPLASTIC THYROID CANCER (ATC) is one of the most aggressive malignancies and often demonstrates resistance to multimodal therapeutic approaches $(1,2)$. The disease occurs rarely, accounting for $1-2 \%$ of thyroid cancers. The rapid progression of ATC to a life threatening status (within a month) is not infrequent, and at initial presentation to a specialist, ATC has often already spread to a systemic disease in many patients. It is thus difficult to treat the patient in a standardized manner, and reliable information about this highly malignant disease is scarce (3). Long-term experiences from single institution often involve different strategies to manage this disease (4-6), and analyses based on large national or multi-institutional databases typically lack the information concerning the details of treatment efforts $(2,7)$. Accurate information concerning the outcomes of treatments for ATC has been exceedingly difficult to establish.

We have established a nationwide research organization, the Anaplastic Thyroid Carcinoma Research Consortium of Japan (ATCCJ), and we assembled the available clinical information into a large database including over a thousand patients with ATC. According to the obtained data, approximately $40 \%$ of patients already have invasive disease that has spread to adjacent organs, and another $40 \%$ of patients have demonstrated disease dissemination to distant $\operatorname{organ}(\mathrm{s})$ at the initial presentation before the initiation of treatment (8). Systemic chemotherapy is therefore indicated in most ATC patients. Several strategies have been employed to control the disease, but conventional chemotherapeutic treatment using doxorubicin or cisplatin did not demonstrate any significant effect $(1,9)$. More recent studies have suggested a potential benefit of taxanes to control advanced disease (10-12), but these small studies used different protocols, and there is currently no reliable objective information concerning the outcomes of a practical standardized treatment plan for ATC patients.

The members of the ATCCJ have addressed this critical situation and planned an investigator-initiated prospective clinica study to determine the present status of the outcomes of a frequently used chemotherapy protocol for patients with ATC (13).

\section{Methods and Patients}

\section{Patients}

The details of the study's concept and protocol have been reported (13). Patients with clinically diagnosed
(RECIST) (14)-defined target lesion was not necessary for study entry to assess the practical treatment outcomes of this rare disease.

The study protocol was approved by the institutional review board of Osaka City University Medical School in March 2012 (\#2248), and then the study was approved by the institutional review board at each of the participating institutions (Supplementary Table S2). The study was performed in accordance with the Declaration of Helsinki. This trial is registered on the clinical trials site of the University Hospital Medical Information Network Clinical Trials Registry Website (UMIN 000008574). All patients were evaluated and treated at the institutions at which they were registered after obtaining written informed consent.

The initial pathology-based diagnoses were made by local pathologists. A central pathology review board was held after the termination of recruitment, by accumulating the tissue specimens from each institution. Three pathologists (described in acknowledgements) who specialize in this field gathered and reviewed every sample to confirm the ATC diagnosis. Confirmation of the pathological diagnosis of ATC was made when all three members agreed. Patients without pathological review data were excluded from final analysis.

\section{Study oversight}

The present study was an investigator-initiated, multicenter, nonrandomized, open-label, single-arm, phase 2 clinical trial to prospectively evaluate the feasibility and efficacy of chemotherapy with weekly paclitaxel administration for patients with ATC (13). The enrolled patients received chemotherapy with a weekly paclitaxel administration $\left[80 \mathrm{mg} / \mathrm{m}^{2}\right.$, once every week (12)]. One course consisted of three paclitaxel administrations (Fig. 1). At least one course of therapy was necessary for each patient. The therapy was continued until the appearance of disease progression or until the time when the patient's physician decided to terminate the protocol. The dose was reduced to $30 \mathrm{mg} / \mathrm{m}^{2}$ when concomitant extra-beam radiation therapy (EBRT) was conducted.

For the evaluation of the treatment's feasibility and safety, we analyzed the relative dose intensity (RDI) and the occurrence of treatment-related adverse events (AE). AEs were reported and graded according to Common Terminology Criteria for Adverse Events v4.0 (15). The RDI was calcu- 4 AU1 lated by the following equation:

$R D I=($ Total dose of paclitaxel administered during the course $) \times 3 /$

(Planned dose administered during the course $) \times($ Total number of weeks necessary for three administrations)

ATC, aged 20 years or older were eligible for this study, if they met the following criteria: Eastern Cooperative Oncology Group performance status of 0 to 2-adequate bone marrow, hepatic, and renal function. The major exclusion criteria included previous chemotherapy or radiation

ST1 therapy for ATC (Supplementary Table S1; Supplementary Data are available online at www.liebertpub.com/thy). Patients of stage IVA to IVC were enrolled and the presence of a Response Evaluation Criteria in Solid Tumors

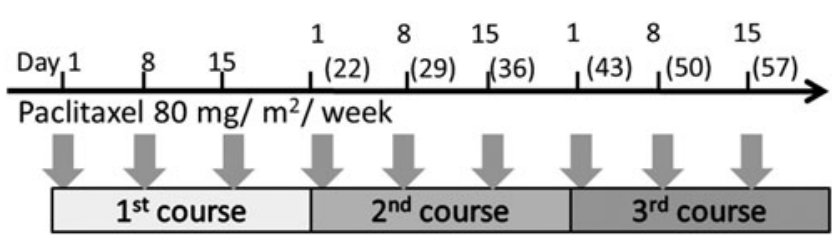

FIG. 1. The schedule of weekly paclitaxel administration $\left(80 \mathrm{mg} / \mathrm{m}^{2}\right)$. 


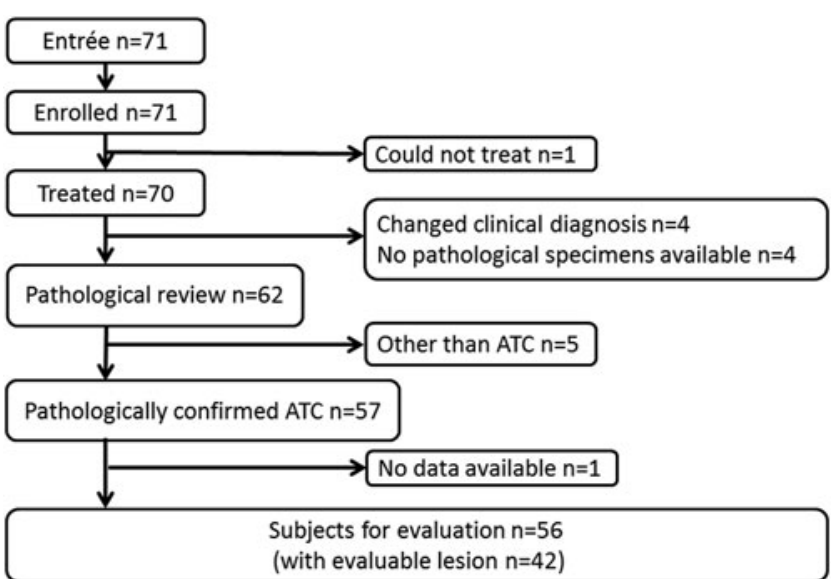

FIG. 2. Cohort diagram. ATC, anaplastic thyroid cancer.

For the analysis of the treatment's efficacy, we determined the overall survival (OS) of all pathologically confirmed ATC patients. The overall response rate, time to progression, and time to treatment failure were evaluated in the patients with RECIST (14)-defined target lesion. The assessments were performed at baseline and every 3 weeks during treatment by computed tomography, and each patient's outcome was classified as a complete response (CR), partial response (PR), stable disease (SD), or progressive disease (PD). The clinical response (CR, PR, or $\mathrm{SD})$ had to be confirmed at least 3 weeks after it was first noted (13).
Initially, 50 patients were set as the target population. This sample size was estimated to be able to provide an evaluation of possible survival benefit. The protocol was then modified to include as many patients as possible until the study period closed (details were described in a previous report) (13). Survival curves are illustrated by the Kaplan-Meier method, and the log-rank test was used to compare the differences in survival. A $p$-value of $<0.05$ was considered significant. The statistical analyses were performed using SPSS 13.0 statistical software (SPSS, Chicago, IL).

\section{Results}

\section{Subjects}

Between April 26, 2012, and March 24, 2014, a total of 71 patients from 14 institutions were enrolled. One patient could not be treated because of the progression of the disease during the evaluation period. The diagnosis of four patients was corrected as disease other than ATC after enrollment. A total of 66 patients thus formed the intended-to-treat series with the study regimen. Specimens for pathological review could not be obtained for four patients, and the remaining 62 patients were evaluated by the central pathology review board, which determined that five patients had thyroid cancers other than ATC. Therefore, $92 \%$ of the pathological diagnoses at the local referral institutions were revealed to be correct after the central review. The final follow-up data could not be obtained for 1 patient. Thus, 56 patients (40 females and 16 males) treated with the study protocol were included in the

Table 1. Baseline Characteristics of the Anaplastic Thyroid Cancer Patients

\begin{tabular}{|c|c|c|c|c|}
\hline Characteristic & Subjects $(\mathrm{n}=56)$ & $(\%)$ & All patients $(\mathrm{n}=71)$ & $(\%)$ \\
\hline \multicolumn{5}{|l|}{ Sex } \\
\hline Female & 40 & (71.4) & 51 & $(71.8)$ \\
\hline Male & 16 & $(28.6)$ & 20 & $(28.2)$ \\
\hline \multicolumn{5}{|l|}{ Age (years) } \\
\hline Range (median) & $47-84(71)$ & & $47-84(71)$ & \\
\hline \multicolumn{5}{|l|}{ Histologic diagnosis } \\
\hline Anaplastic thyroid cancer & 56 & $(100)$ & 57 & $(80.1)$ \\
\hline Papillary carcinoma & - & - & 4 & $(5.6)$ \\
\hline Squamous cell carcinoma & - & - & 1 & (1.4) \\
\hline Others & - & _- & 4 & $(5.6)$ \\
\hline Not evaluable & - & - & 5 & $(7.0)$ \\
\hline \multicolumn{5}{|l|}{ Clinical stage } \\
\hline IVA & 11 & (19.6) & 11 & $(15.5)$ \\
\hline IVB & 19 & (33.9) & 26 & (36.6) \\
\hline IVC & 22 & $(39.3)$ & 28 & $(39.4)$ \\
\hline $\mathrm{X}$ & 4 & $(7.1)$ & 6 & $(8.5)$ \\
\hline \multicolumn{5}{|c|}{ Eastern Cooperative Oncology Group performance status } \\
\hline 0 & 46 & $(82.1)$ & 57 & $(80.3)$ \\
\hline 1 & 8 & $(14.3)$ & 9 & $(12.7)$ \\
\hline 2 & 2 & $(3.6)$ & 5 & $(7.0)$ \\
\hline Prior surgery for anaplastic thyroid cancer & 25 & $(44.6)$ & 27 & $(38.0)$ \\
\hline Resectable & 18 & $(32.1)$ & 20 & $(28.2)$ \\
\hline Palliative surgery or biopsy & 7 & $(12.5)$ & 7 & $(9.6)$ \\
\hline \multicolumn{5}{|l|}{ Target lesion } \\
\hline Primary tumor & 30 & $(53.6)$ & 40 & $(56.3)$ \\
\hline Locally recurrent tumor & 6 & $(10.7)$ & 9 & (12.7) \\
\hline Metastatic lesion & 6 & $(10.7)$ & 8 & (11.3) \\
\hline None & 14 & $(25.0)$ & 14 & $(19.7)$ \\
\hline
\end{tabular}


Table 2. Treatment-Related Adverse Events

\begin{tabular}{|c|c|c|c|c|}
\hline Event & All grades & $(\%)$ & Grade 3 and over & $(\%)$ \\
\hline \multicolumn{5}{|l|}{ All treatment-related adverse events } \\
\hline Adverse events (AEs) & 55 & $(98.2)$ & 16 & $(28.6)$ \\
\hline Severe AEs & 0 & $(0)$ & 0 & $(0)$ \\
\hline \multicolumn{5}{|l|}{ Treatment-related AEs } \\
\hline \multicolumn{5}{|l|}{ Hematological AE } \\
\hline Anemia & 44 & (76.8) & 3 & $(5.4)$ \\
\hline Leukocytopenia & 27 & $(48.2)$ & 5 & $(8.9)$ \\
\hline Neutropenia & 26 & $(46.4)$ & 6 & $(10.7)$ \\
\hline Hypoalbuminemia & 25 & (44.6) & 0 & (0) \\
\hline AST/ALT elevation & 19 & (33.9) & 1 & $(1.8)$ \\
\hline Hypercalcemia & 4 & $(7.1)$ & 1 & $(1.8)$ \\
\hline Thrombocytopenia & 1 & $(1.8)$ & 0 & $(0)$ \\
\hline Creatinine elevation & 1 & $(1.8)$ & 0 & $(0)$ \\
\hline \multicolumn{5}{|l|}{ Nonhematological AEs } \\
\hline Alopecia & 38 & $(67.9)$ & - & - \\
\hline Fatigue & 27 & (48.2) & 0 & $(0)$ \\
\hline Peripheral neuropathy & 16 & (28.6) & 1 & $(1.8)$ \\
\hline Nausea & 13 & $(23.2)$ & 0 & $(0)$ \\
\hline Constipation & 10 & (17.9) & 0 & $(0)$ \\
\hline Dyspnea & 11 & (19.6) & 4 & $(7.1)$ \\
\hline Rush & 9 & (16.1) & 2 & $(3.6)$ \\
\hline Mucositis oral & 8 & (14.3) & 1 & $(1.8)$ \\
\hline Fever & 5 & $(8.9)$ & 1 & $(1.8)$ \\
\hline Vomiting & 3 & $(5.4)$ & 0 & $(0)$ \\
\hline Pneumonitis & 2 & (3.6) & 0 & $(0)$ \\
\hline Complete atrioventricular block & 1 & (1.8) & 1 & $(1.8)$ \\
\hline Diarrhea & 1 & $(1.8)$ & 0 & $(0)$ \\
\hline Infection & 1 & $(1.8)$ & 0 & $(0)$ \\
\hline Edema & 1 & $(1.8)$ & 0 & (0) \\
\hline Phlebitis, allergic reaction, febrile neutropenia & 0 & $(0)$ & 0 & (0) \\
\hline
\end{tabular}

feasibility and survival analysis. Among these 56 patients, 42 had RECIST (14)-defined target lesion, and these cases form the cohort for the efficacy analysis (Fig. 2). The baseline characteristics of the patients are summarized in Table 1 .

\section{Feasibility assessment}

Fifty-two of the 56 patients $(92.9 \%)$ were successfully treated with more than one course of the protocol. Four patients could not complete a single course of treatment because of complete atrioventricular block (one case), rapid progression of the disease (one case), or patient refusal (two cases). Two to 69 administrations of paclitaxel (0 to 23 courses) were conducted with a median number of six administrations (2 courses). The protocol treatment had to be postponed in 29 patients: in 12 patients during the first course, 11 patients during their second course, 1 patient in the third course, 2 during the fourth course, 1 during the fifth course, and 2 patients during a later course of the treatment. The median time to treatment failure was 1.6 months [confidence interval (CI)

ST3 0.9-2.3]. The RDI in every course is shown in Supplementary Table S3. The average RDI of more than $80 \%$ could be maintained during the initial two courses of the treatment.

No death or severe AE occurring during the treatment was T2 reported. The AEs that were reported are listed in Table 2. A total of 55 patients $(98.2 \%)$ had at least one treatment-related $\mathrm{AE}$ during the protocol treatment. The most commonly reported AEs of all grades were anemia $(78.6 \%)$ and alopecia (67.9\%). AEs of grade 3 or higher were observed in 16 patients
(28.6\%); these included neutropenia (10.7\%), leukocytopenia $(8.9 \%)$, dyspnea $(7.1 \%)$, anemia $(5.4 \%)$, rash $(3.6 \%)$, liver dysfunction (1.8\%), fever (1.8\%), mucositis $(1.8 \%)$, peripheral neuropathy $(1.8 \%)$, hypercalcemia $(1.8 \%)$, and complete atrioventricular block (1.8\%).

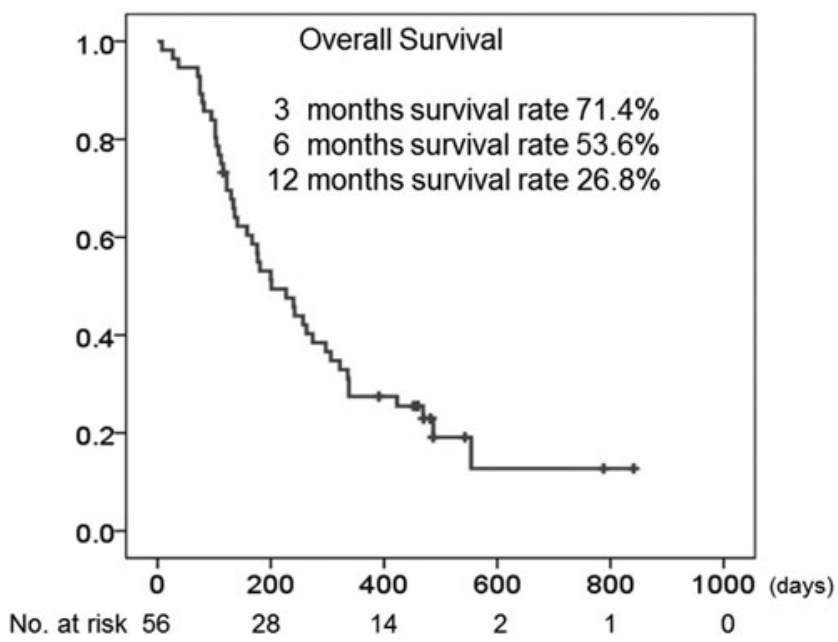

FIG. 3. Overall survival plot of all 56 patients confirmed to have ATC pathologically determined by the KaplanMeier method. Fifty patients were treated with chemotherapy alone, and six patients were treated with chemotherapy and concomitant radiation therapy. 


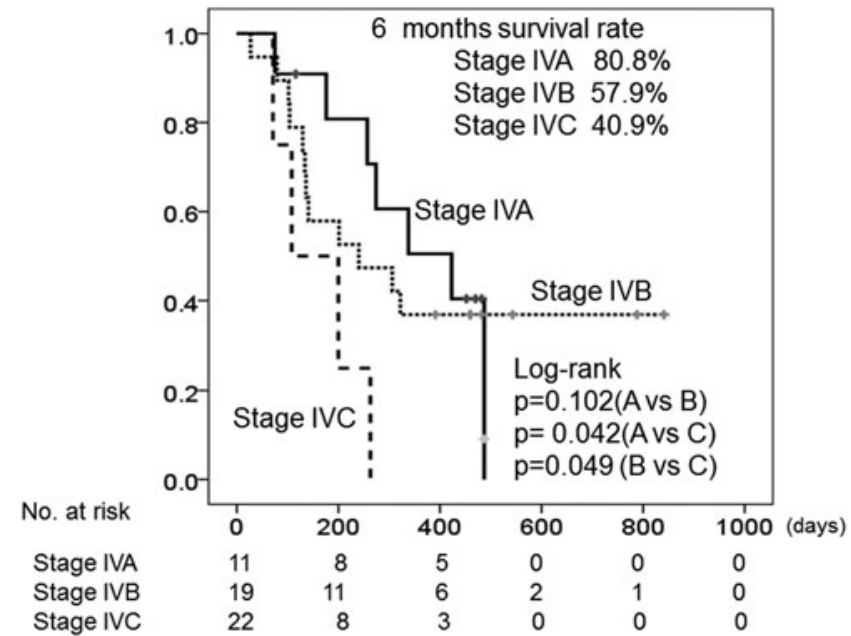

FIG. 4. Overall survival plot and 6-month survival rates determined by the Kaplan-Meier method, stratified by the clinical stage (IVA to IVC) of the disease. Four out of all 56 patients with anaplastic thyroid cancer in the locally recurrent disease of previously treated thyroid cancer group were excluded from stage classification.

\section{Efficacy assessment}

Forty-four patients died (43 died of disease and one in an accident), and 11 patients remained alive for 15.3-26.3 months with a median of 16.1 months at the time of the data cutoff. The median OS of all 56 ATC patients was 6.7 months

F3 [CI 4.4-9.0] (Fig. 3). The 3-, 6-, and 12-month OS rates were $71.4 \%, 53.6 \%$, and $26.8 \%$, respectively. The median causespecific survival was 6.7 months [CI 4.1-9.3].

The median OS for the patients with stage IVA, IVB, and IVC disease were 14.1 [CI 6.6-21.6], 8.0 [CI 0.2-15.8], and 5.3 [CI 3.4-7.1] months, respectively. The 6-month survival rates were $80.8 \%, 57.9 \%$, and $40.9 \%$ for stage IVA, IVB, and IVC patients, respectively. The patients classified as having stage IVC disease showed significantly poorer survival compared to those with stage IVA or IVB disease (log-rank F4 test $p=0.042$ or 0.049 ; Fig. 4).

Although a measurable target lesion was identified in 42 patients, 3 patients could not complete a single course of the protocol treatment. Of the 42 evaluable tumors, 21 (50.0\%) showed a variable extent of tumor shrinkage (Fig. 5). No patient demonstrated a CR or durable SD (i.e., SD persisting for $>3$ months). Nine, twenty-two, and eight of these 42 patients $(21 \%, 52 \%$, and $19 \%)$ demonstrated PR, SD, and PD, respectively. An objective response $(\mathrm{CR}+\mathrm{PR})$ was thus confirmed in $9(21.4 \%)$ patients, and a clinical benefit (CR + $\mathrm{PR}+\mathrm{SD})$ was observed in $31(73.8 \%)$ patients. The time to progression was $0.4-26.3$ months (median 1.6 months). Three patients received concomitant EBRT.

Overall, EBRT was conducted in six patients with a dose of 40 (three patients) or 60 (three patients) Gy. A patient with stage IVA disease and two patients with stage IVB disease received EBRT as an adjuvant therapy after curative surgery and survived 10.7 to 28.1 months. Two other patients with stage IVC disease received EBRT for the primary neck tumor as a part of systemic therapy; they showed PR or SD and survived 3.3 and 11.3 months, respectively. Another patient received EBRT for a lung metastasis after locally curative operation for stage IVA disease and survived 9.1 months with confirmed SD. The median OS of these patients were longer (10.7 months [CI 8.2-13.3]) than those who did not receive EBRT (5.9 months [CI 4.2-7.6]) but no statistical difference was identified (log-rank test $p=0.204$ ). The study protocol was initially conducted as neo-adjuvant chemotherapy in 14 patients. Of these, 2, 8, and 4 patients were classified to have stage IVA, IVB, and IVC disease, respectively. Complete removal of the local tumor could be achieved in 8 patients $(5$ patients with stage IVB and 3 with stage IVC disease) after chemotherapy. They survived from 3.3 to 26.3 months, and that was significantly longer (median 7.6 months [CI 8.123.0]) than the other 34 patients (5.4 months [CI 3.0-7.8], $p=0.018$ ) (Fig. 6). Of these 34 patients, 4, were classified as having stage IVA, 9 as IVB, 17 as IVC, and 4 as having stage $\mathrm{X}$ disease.

\section{Discussion}

Several observations suggested that taxanes might be beneficial agents for controlling ATC (3,10-12). For example, Ain et al. demonstrated the efficacy of paclitaxel in 19 patients with ATC for the first time in 2000; they administered a 96-hour continuous infusion of the agent in a 21-day

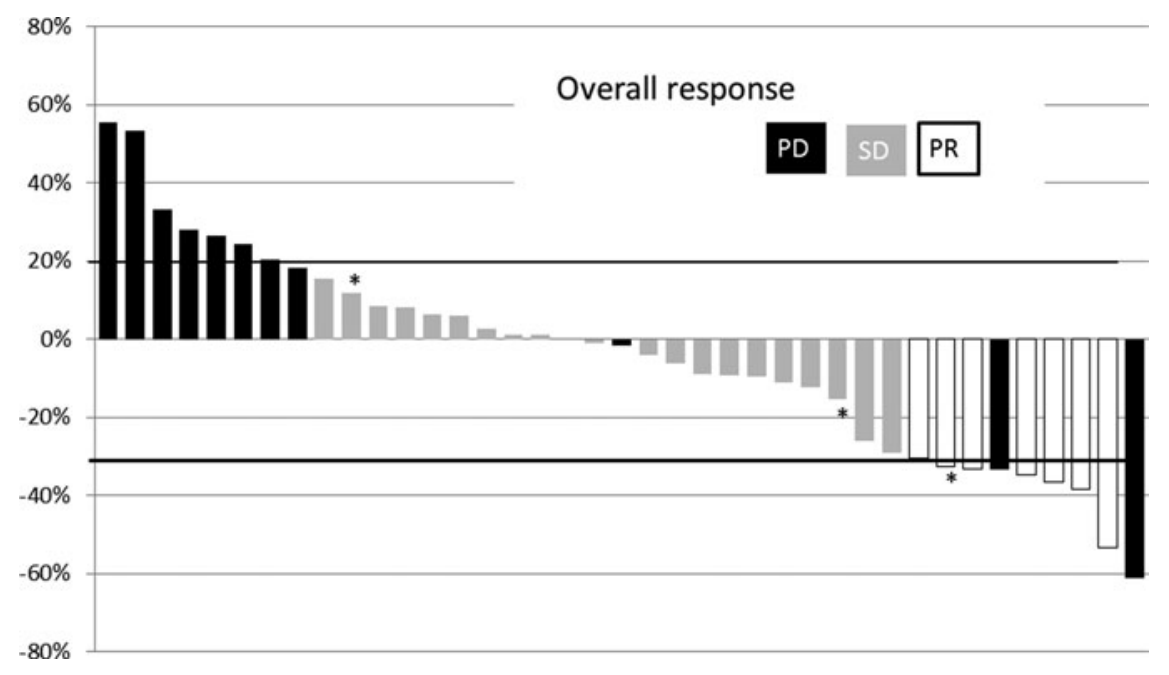

FIG. 5. Waterfall plot showing the effect of the treatment on Response Evaluation Criteria in Solid Tumorsdefined target lesion. The result of 39 patients who accomplished at least one course of protocol treatment was demonstrated within the cohort of 42 patients with evaluable lesion. Patients who received concomitant radiation therapy were included $(*)$. 


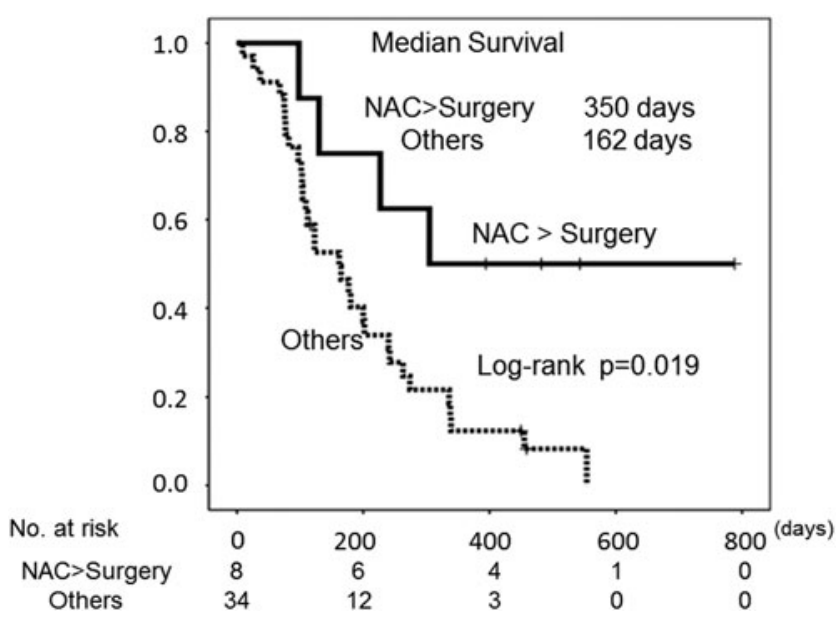

FIG. 6. Cause-specific survival plot determined by the Kaplan-Meier method. Survival was compared between the patients whose tumor has completely removed after neoadjuvant chemotherapy (NAC; $n=8$ ), and other patients in whom the tumor could not be completely removed (including no surgery) after chemotherapy $(n=34)$ in the cohort for efficacy analysis.

cycle, and the results demonstrated a response rate of 53\%, with one CR patient (10). Kawada et al. used docetaxel in a 21-day cycle for 7 ATC patients, and they reported a response rate of $43 \%$ with one CR (11). Higashiyama et al. reported their retrospective experience with a weekly paclitaxel administration in 13 patients: a response rate of $31 \%$ with one CR was observed. They concluded that weekly administration of paclitaxel could be beneficial to extend the survival of ATC patients with stage IVB disease (12). Our present findings objectively confirm the feasibility and efficacy of paclitaxel-based chemotherapy in ATC patients. Our study also demonstrates the potential usefulness of weekly paclitaxel treatment as a neo-adjuvant chemotherapy for patients with ATC, as suggested by Higashiyama et al. (12).

Haymart et al. reported a median OS of stage IVA, IVB, and IVC ATC patients as 9.0, 4.8, and 3.0 months, respectively, by analyzing a large cohort of a national cancer database (2). Our results demonstrate much longer OS of 14.1, 8.0, and 5.3 months, respectively. A clinical trial by Sosa et al. investigating the efficacy of fosbretabulin in addition to a combination protocol with paclitaxel and carboplatin enrolled mainly patients with stage IVC metastatic ATC (16). The results presented here concerning the survival of stage IVC patients (median OS 5.3 months; 6-month survival $40.9 \%$ ) seems better compared to the control arm of the study by Sosa et al. (OS 4.0 months, $34.9 \%$ ), and they are in line with the results of the fosbretabulin arm (OS 5.2 months, $48.1 \%$ ). Other studies reported that the median survival of ATC patients with stage IVC disease was 2.5 (6) or 2.7 months (8). However, it is difficult to compare these outcomes due to differences in eligibility and treatment approaches in each study.

We could not exclude the administration of EBRT in this study, because radiation was the only recommended treatment stated in the Japanese guideline for ATC at the time when the study started (9). The OS of the six patients receiving EBRT was longer, though it was not significantly different and it may, at least in part, be caused by patient selection bias. Radiation therapies were performed as an adjuvant therapy after surgical removal of the tumor in three patients. Several previous studies have demonstrated a benefit of EBRT to control local ATC disease (17-19). Further structured studies are required to further clarify the role and significance of EBRT.

All the AEs observed in the present study were tolerable and manageable. The results of our study confirm that the protocol used could be performed without changes and with a sufficient dose intensity during early courses. This protocol might also be conducted in an outpatient setting. We were also able to terminate the protocol, when necessary, without any prolonged AEs that would delay the start of treatment following chemotherapy or AEs that would impair patients' quality of life.

The progress of the patient enrollment was much smoother than had been expected (13). There was no patient who did not meet the criteria at the initial eligibility evaluation and no protocol violation during the course to exclude a patient from the study. In addition, $92 \%$ of the pathological diagnoses at local referral institutions were confirmed to be correct. These substantial baseline qualities of the registered institutions were clearly due to the concordant desire and coordination of the ATCCJ study group members. The lack of a standardized therapeutic strategy and the simple protocol used in this study contributed positively to enroll patients for active participation in this study.

The treatment protocol presented here can be used for ATC patients, and it showed marginal clinical benefit. In conclusion, this study clearly demonstrates the feasibility and objective outcomes of a standardized chemotherapy by weekly paclitaxel for ATC patients.

\section{Acknowledgments}

We thank all of the patients who participated in this study. The study was approved and supported by all of the members of ATCCJ. The authors sincerely thank Professor Yoshihide Fujimoto and Professor Michio Sowa for reviewing and monitoring the study as members of the safety review board, and Dr. Mitsuyoshi Hirokawa, Professor Kennichi Kakudo, and Professor Atsuhiko Sakamoto for reviewing the tumor samples as members of Central Pathology Review Board. This study was initiated with thoughtful support from Professor Kosei Hirakawa of Osaka City University. We also thank Ms. Yuka Matsumoto and the members of the Center for Drug and Food Clinical Evaluation of Osaka City University Hospital for the data accumulation and management.

This work was supported in part by a grant-in-aid for scientific research from the Japan Society for the Promotion of Science ( JSPS KAKENHI \#25461992) to N.O., a grant-inaid for strategic research from Osaka City University (Novel Priority Research B) to N.O., and a study grant from the nonprofit organization TODoc Network.

\section{Author Disclosure Statement}

N.O. received honoraria and research funding from Eisai and Bayer and is a member of the advisory boards of Eisai and Bayer. I.S. received honoraria from Eisai and Bayer; research funding from Eisai; travel expenses from Bayer, Eisai, Genzyme; and is a member of advisory board of AstraZeneca and Bayer. All remaining authors have declared no conflicts of interest. 


\section{References}

1. Smallidge RC 2012 Approach to the patient with anaplastic thyroid carcinoma. J Clin Endocrinol Metab 97:2566-2572.

2. Haymart MR, Banerjee M, Yin H, Worden F, Griggs JJ 2013 Marginal treatment benefit in anaplastic thyroid cancer. Cancer 119:3133-3139.

3. Smallridge RC, Ain KB, Asa SL, Bible KC, Brierley JD, Burman KD, Kebebew E, Lee NY, Nikiforov YE, Rosenthal MS, Shah MH, Shaha AR, Tuttle RM 2012 American Thyroid Association Anaplastic Thyroid Cancer Guidelines Taskforce: American Thyroid Association guidelines for management of patients with anaplastic thyroid cancer. Thyroid 22:1104-1139.

4. McIver B, Hay I, Giuffrida D, Dvorak CE, Grant CS, Thompson GB, van Heerden JA, Goellner JR 2001 Anaplastic thyroid carcinoma: a 50-year experience at a single institution. Surgery 130:1028-1034.

5. Akaishi J, Sugino K, Kitagawa W, Nagahama M, Kameyama K, Shimizu K, Ito K, Ito K 2011 Prognostic factors and treatment outcomes of 100 cases of anaplastic thyroid carcinoma. Thyroid 21:1183-1189.

6. Foote RL, Molina JR, Kasperbauer JL, Lloyd RV, McIver B, Morris JC, Grant CS, Thompson GB, Richards ML, Hay ID, Smallridge RC, Bible KC 2011 Enhanced survival in locoregionally confined anaplastic thyroid carcinoma: a single-institution experience using aggressive multimodal therapy. Thyroid 21:25-30.

7. Kebebew E, Greenspan F, Clark O, Woeber KA, McMillan A 2005 Anaplastic thyroid carcinoma treatment outcome and prognostic factors. Cancer 103:1330-1335.

8. Sugitani I, Miyauchi A, Sugino K, Okamoto T, Yoshida A, Suzuki S 2012 Prognostic factors and treatment outcomes for anaplastic thyroid carcinoma: ATC Research Consortium of Japan cohort study of 677 patients. World J Surg 361:247-1254.

9. Sugino K \& Onoda N 2010 Part VII. Anaplastic carcinoma. In: Takami H, Ito Y, Noguchi H, Yoshida A, Okamoto T (eds) Treatment of Thyroid Tumor: Japanese Clinical Guidelines. Springer Tokyo, Tokyo, pp 201-216.

10. Ain KB, Egorin MJ, DeSimone PA 2000 Treatment of anaplastic thyroid carcinoma with paclitaxel: phase 2 trial using ninety-six-hour infusion. Collaborative Anaplastic Thyroid Cancer Health Intervention Trials (CATCHIT) Group. Thyroid 10:587-594.

11. Kawada K, Kitagawa K, Kamei S, Inada M, Mitsuma A, Sawaki M, Kikumori T, Fujimoto Y, Arima H, Imai T, Ando Y 2010 The feasibility study of docetaxel in patients with anaplastic thyroid cancer. Jpn J Clin Oncol 40:596599.
12. Higashiyama T, Ito $\mathrm{Y}$, Hirokawa $\mathrm{M}$, Fukushima $\mathrm{M}$, Uruno T, Miya A, Matsuzuka F, Miyauchi A 2010 Induction chemotherapy with weekly paclitaxel administration for anaplastic thyroid carcinoma. Thyroid 20:7-14.

13. Onoda N, Sugitani I, Higashiyama T, Hara H, Ito K, Kammori M, Sugino K, Suzuki S, Toda K, Yoshida A, Miyauchi A 2015 Concept and design of a nationwide prospective feasibility/efficacy/safety study of weekly paclitaxel for patients with pathologically confirmed anaplastic thyroid cancer (ATCCJ-PTX-P2). BMC Cancer 20:475.

14. Eisenhauer EA, Therasse P, Bogaerts J, Schwartz LH, Sargent D, Ford R, Dancey J, Arbuck S, Gwyther S, Mooney M, Rubinstein L, Shankar L, Dodd L, Kaplan R, Lacombe D, Verweij J 2009 New response evaluation criteria in solid tumours: revised RECIST guideline (version 1.1). Eur J Cancer 45:228-247.

15. National Cancer Institute 2009 Common Terminology Criteria for Adverse Events (CTCAE) version 4.0. Bethesda, MD: National Cancer Institute. Available at: http:// evs.nci.nih.gov/ftp1/CTCAE/CTCAE_4.03_2010-0614_QuickReference_5x7.pdf (accessed February 8, 2015).

16. Sosa JA, Elisei R, Jarzab B, Balkissoon J, Lu SP, Bal C, Marur S, Gramza A, Yosef RB, Gitlitz B, Haugen BR, Ondrey F, Lu C, Karandikar SM, Khuri F, Licitra L, Remick SC 2014 Randomized safety and efficacy study of fosbretabulin with paclitaxel/carboplatin against anaplastic thyroid carcinoma. Thyroid 24:232-240.

17. Troch M, Koperek O, Scheuba C, Dieckmann K, Hoffmann M, Niederle B, Raderer M 2010 High efficacy of concomitant treatment of undifferentiated (anaplastic) thyroid cancer with radiation and docetaxel. J Clin Endocrinol Metab 95:E54-57.

18. Tanaka K, Sugitani I, Fujimoto Y 2011 A novel chemoradiotherapy with low-dose daily cisplatin, 5-fluorouracil and doxorubicin for anaplastic thyroid carcinoma: a preliminary report. Jpn J Clin Oncol 41:1074-1078.

19. Onoda N, Kashiwagi S, Noda S, Kawajiri H, Takashima T, Ishikawa T, Hirakawa K 2013 High efficacy of chemoradiation therapy sensitized by weekly docetaxel for anaplastic thyroid cancer. Anticancer Res 33:3445-3448.

Address correspondence to:

Naoyoshi Onoda, MD, PhD

Department of Surgical Oncology

Osaka City University Graduate School of Medicine

1-4-3 Asahi-machi

Abeno-ku, Osaka 545-8585

Japan

E-mail: nonoda@med.osaka-cu.ac.jp 


\section{Supplementary Data}

Supplementary Table S1. Patient Eligibility Criteria

Inclusion criteria

Patient who fulfilled all of the following criteria were included:

(1) Pathologically proven anaplastic thyroid cancer

(2) Age $\geq 20$ years

(3) Eastern Cooperative Oncology Group performance status 0-2

(4) Biochemical parameters and electrocardiogram
(A) Leucocyte count $\geq 3000 / \mathrm{mm}^{3}$
(B) Neutrophil count $\geq 1500 / \mathrm{mm}^{3}$
(C) Platelet count $\geq 100,000 / \mathrm{mm}^{3}$

(D) Hemoglobin $\geq 9.5 \mathrm{~g} / \mathrm{dL}$

AU3 $\quad$ (E) AST(GOT), ALT(GPT) $\leq$ two times the facility's standard

(F) Total bilirubin $\leq 1.5 \mathrm{mg} / \mathrm{dL}$

(G) Serum creatinine $\leq 1.5 \mathrm{mg} / \mathrm{dL}$

(H) Creatinine clearance $\geq 50 \mathrm{~mL} / \mathrm{min}$

(I) No severe abnormality on electrocardiogram

(5) Signed written informed consent

\section{Exclusion criteria}

Patients who had one or more of the following criteria were excluded:

(1) Suspicion of interstitial pneumonia or pulmonary fibrosis by chest radiograph

(2) Brain metastasis with symptoms

(3) Presence of active peptic ulcer

(4) Presence of active other malignancies

(5) Fever $>38^{\circ} \mathrm{C}$

(6) Severe complication (e.g., myocardial infarction within 3 months, heart failure, poorly controlled diabetes mellitus, severe infectious disease)

(7) History of severe drug allergy

(8) History of hypersensitivity to polyoxyethylated or hydrogenated castor oil

(9) Hypersensitivity to alcohol

(10) Pregnant or nursing women

(11) Psychological problems

(12) Previous chemotherapy or radiation therapy for the present illness

(13) Physician has judged the patient inappropriate for entry in this trial 
Supplementary Table S2. Institutions and Representatives Who Participated in This Clinical Study

\begin{tabular}{ll}
\hline Institute & Representative \\
\hline Dept. of Surgical Oncology, Osaka City University Graduate School of Medicine & Naoyoshi Onoda \\
Dept. of Surgery, Kuma Hospital & Takuya Higashiyama \\
Dept. of Surgery, Kanaji Hospital & Tatsuya Fukumori \\
Dept. of Surgery, Ito Hospital & Kiminori Sugino \\
Dept. of Surgery II, Shinshu University & Kazuhisa Toda \\
Division of Head and Neck, Cancer Institute Hospital & Takaaki Yamanouchi \\
Dept. of Surgery, Nagasaki Univ. Grad. Sch. of Biomedical Science & Akira Yoshida \\
Dept. of Breast and Endocrine Surgery, Kanagawa Cancer Ctr. & Hisato Hara \\
Dept. of Breast and Endocrine Surgery, Tsukuba Univ. & Shinichi Suzuki \\
Dept. of Thyroid and Endocrinology, Fukushima Med. Univ. Sch. of Med. & Shigeto Maeda \\
Dept. of Surgery, National Hospital Org. Nagasaki Medical Ctr. & Noriaki Nakashima \\
Dept. of Breast and Endocrine Surgery, Tohoku Univ. & Hitoshi Noguchi \\
Noguchi Thyroid Clinic and Hospital Foundation & Nobuhiro Hanai \\
Dept. of Head and Neck Surgery, Aichi Cancer Ctr. Hospital & Koki Miura \\
Dept. of Surg., International Univ. of Health and Welfare, Mita Hospital & Kiyoshi Shingu \\
Dept. of Breast and Endocrine Surgery, Iida Municipal Hospital & Masanori Koizumi \\
Dept. of Surgery, Mito Medical Ctr. & Shigeto Yamagata \\
Dept. of Surgery, Sumitomo Hospital & Kiyoaki Tsukahara \\
Dept. of Otolaryngology, Head \& Neck Surgery, Tokyo Med. Univ. Hachioji Med. Ctr. & Toshimitsu Nemoto \\
Dept. of Otorhinolaryngology, Japanese Red Cross Narita Hospital & Seiichi Yoshimoto \\
Dept. of Head and Neck Oncology, National Cancer Ctr. Hospital & Yuki Tomizawa \\
Dept. of Surgery, Uchimaru Hospital & Kiyoto Shiga \\
Dept. of Otorhinolaryngology, Iwate Medical Univ. & Nobuyasu Suganuma \\
Dept. of Surgery, Yokohama City Univ. & Katsuhiko Masudo \\
Dept. of Breast and Endocrine Surg., Yokohama City Univ. Med. Ctr. & Toshinari Takamura \\
Dept. of Endocrinology and Metabolism, Kanazawa Univ. Hospital & Iwao Sugitani \\
Dept. of Endocrine Surgery, Nippon Medical School & Hiroyuki Tsuji \\
Dept. of Head and Neck Surgery, Kanazawa Medical Univ. & \\
\hline
\end{tabular}


THY-2016-0072-ver9-Onoda-Suppl_1P.3d 08/22/16 7:27pm Page 3

Supplementary Table S3. Relative Dose Intensity Administered in Each Course

\begin{tabular}{lccc}
\hline & \multicolumn{2}{c}{ No. of patients treated } & $\begin{array}{c}\text { Average } \\
\text { relative dose } \\
\text { intensity }\end{array}$ \\
\cline { 2 - 4 } No. of courses & Intended & Accomplished (\%) & \\
\hline 1 & 56 & $52(92.9 \%)$ & 92.0 \\
2 & 42 & $35(83.3 \%)$ & 84.9 \\
3 & 27 & $17(63.0 \%)$ & 74.1 \\
4 & 15 & $12(80.0 \%)$ & 72.8 \\
$5-7$ & 15 & $13(86.7 \%)$ & 72.1 \\
$8-23$ & 21 & $19(90.5 \%)$ & 70.7 \\
\hline
\end{tabular}




\section{AUTHOR QUERY FOR THY-2016-0072-VER9-ONODA_1P}

AU1: Citation number changed to 15 to match reference for Common Terminology Criteria for Adverse Events. Please ensure this is correct.

AU2: Please confirm year of publication.

AU3: Please expand abbreviated terms. 